\title{
Quantum Entanglement from Positronium Decay
}

\author{
Forrest Bullard ${ }^{1}$ \\ ${ }^{1}$ California State University, Chico
}

December 14, 2020

\section{Introduction}

In this experiment we will explore whether photons emitted from the decay of positronium, a positron-electron composition, are quantum entangled. When talking about quantum entangled particles it is impossible to fully represent the quantum state of one particle independently of another such that if we know one photon from an entangled pair is horizontally polarized then the other must be vertically polarized. We will use this concept along with knowledge from previous experiments on positron-electron annihilation and Compton scattering to try to determine the truth of this model.

\section{Procedure}

In this experiment we used two NaI detectors, photo-multiplier tubes (PMT), pre-amplifiers, and amplifiers. Along with two single channel analyzers, a logic gate, a coincidence detector, a high-voltage supply, and a multi-channel analyzer (MCA) and of course two aluminum targets, a sodium-22 radioactive source, and loads of lead bricks. Our source was encased in the center of our detector configuration such that radiation from the source was focused towards both aluminum targets placed opposite of each other with respect to the source. Both detectors were then stationed so that photons coming from the source were detected after a 90 degree Compton scattering from the target. This ninety degree correlation is necessary for proof of quantum entanglement as we expect no coincidences to occur when both detectors are in the same plane do to quantum pairs anti-correlated polarization causing expected scattering to be related by this ninety degree angle. The picture below shows the basic geometry of this set up. 


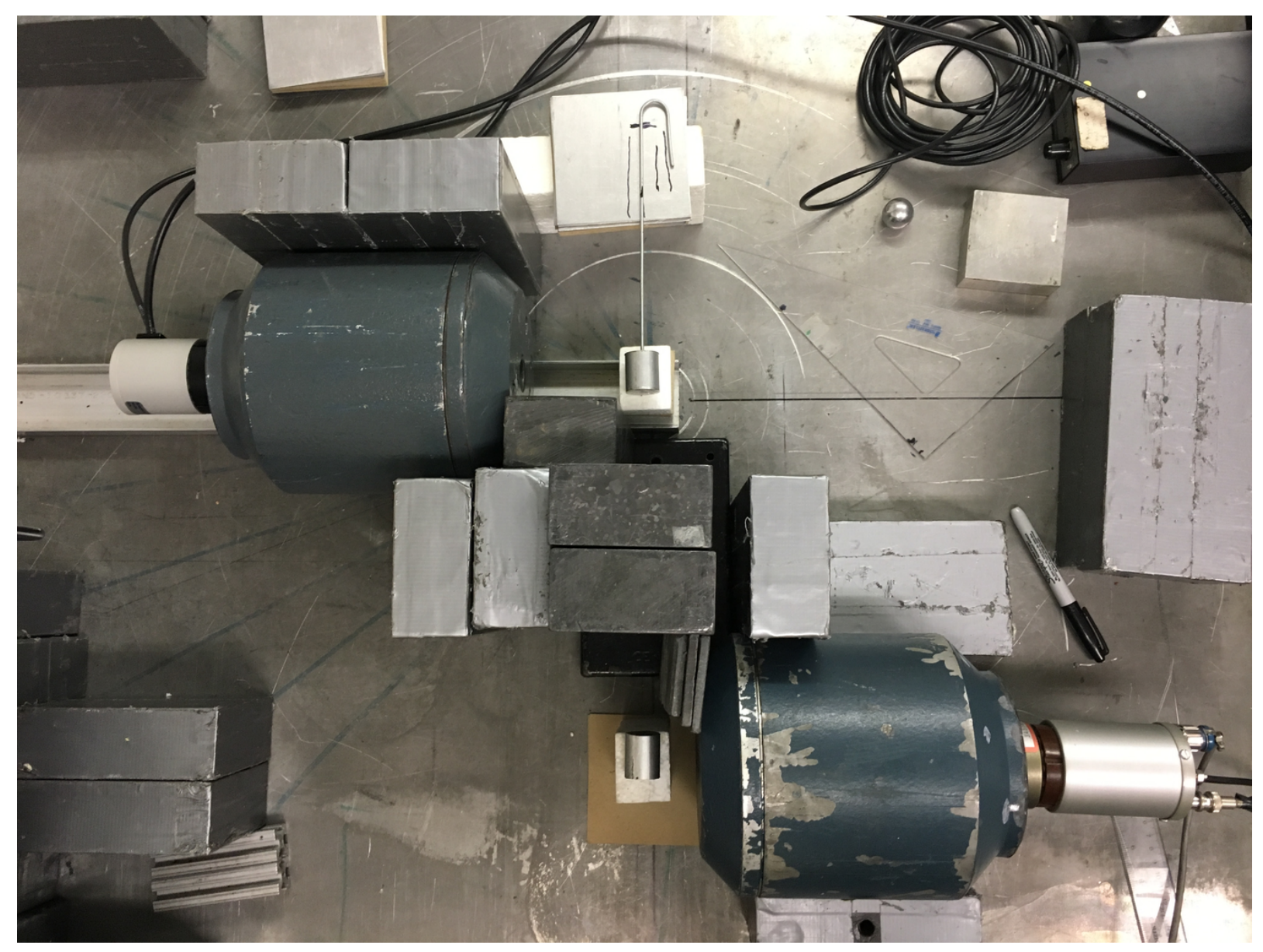

Figure 1: Detector 2 (D2) top left and Detector 1 (D1) bottom right

It was also necessary to move detector one into an upright position (directly above the target) as this is the position we expect to get full coincidences. The MCA was used to make sure that coincidences were were only counted for photons paired with a Compton scattered 0.511 $\mathrm{MeV}$ photon, as well as to check for background noise and to compare full spectrum of both detectors.

\section{Data}

Full spectrum runs were done at two hour interval for both detectors to check that photons were being detected at proper energy intervals as would be expected for $0.511 \mathrm{MeV}$ photons being Compton scattered from a target at 90 degrees. 


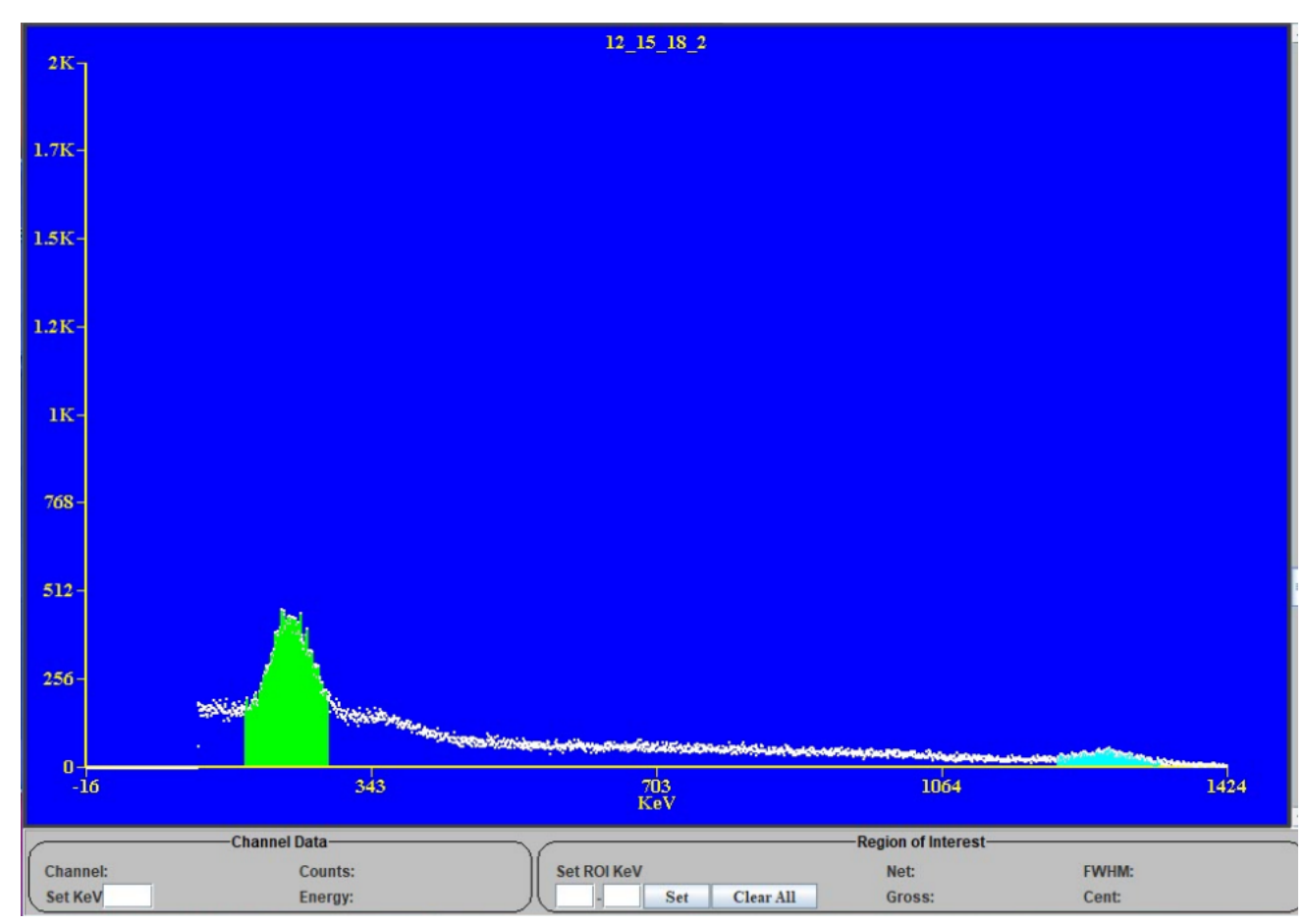

Figure 2: Full Spectrum D1

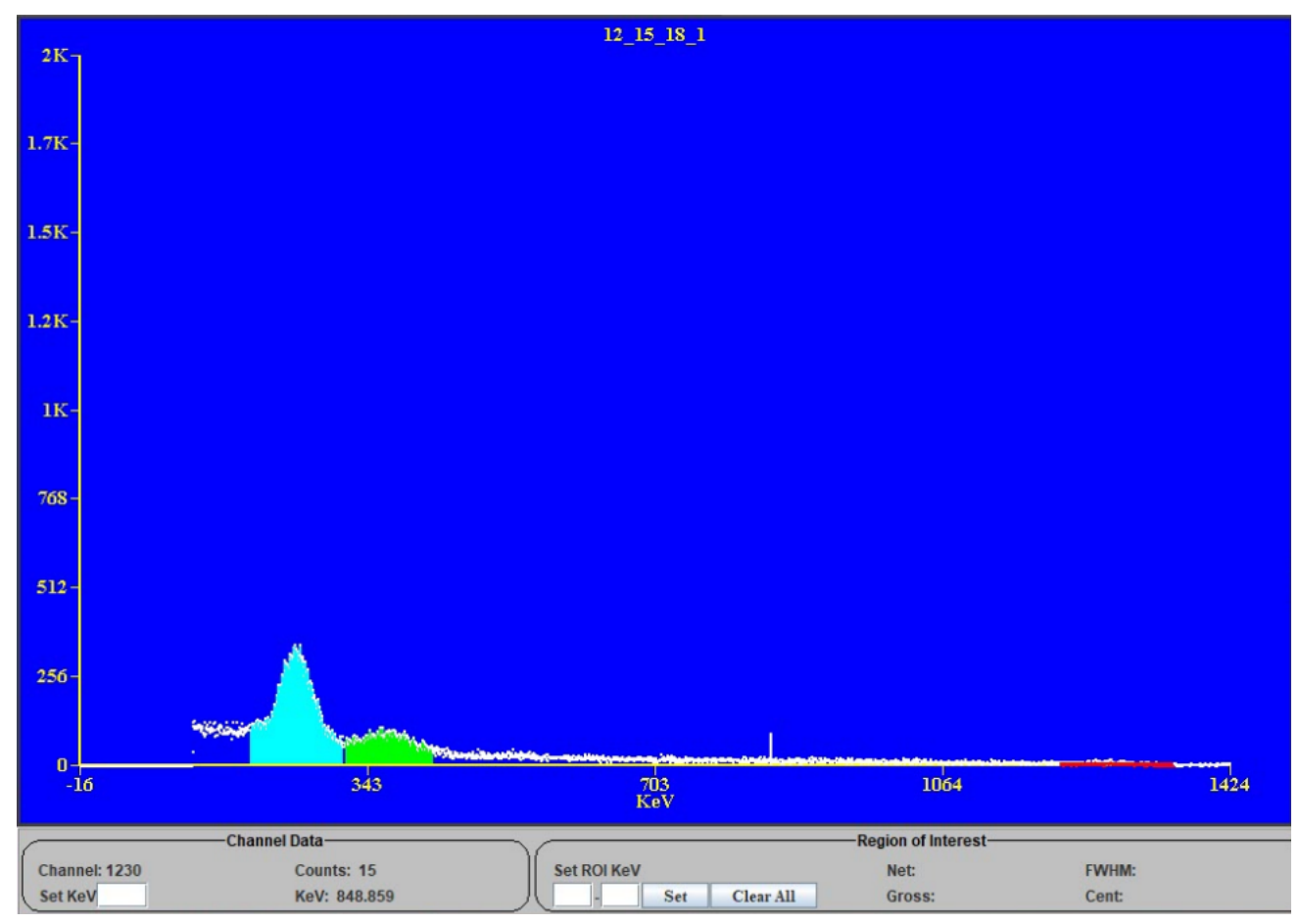

Figure 3: Full Spectrum D2 
The first peak from the left in both MCA spectrum is approximately the appropriate energy for 90 degree $0.511 \mathrm{MeV}$ scattering which we expect to have an energy of $0.255 \mathrm{MeV}$. The peak in D1 has a centered energy of $0.238 \pm 0.047 \mathrm{MeV}$ and the peak in D2 has a centered energy of $0.252 \pm 0.039 \mathrm{MeV}$ both within reason. The second smaller peak which is easier to make out in the spectrum of D2 is also in line with Compton scattering we expect to see for a $1.27 \mathrm{MeV}$ photon an unfortunate byproduct of the decay of sodium-22 which will not be quantum entangled with the $0.511 \mathrm{MeV}$ photons being examined but effects our results none-the-less. One final thing to note is the reduction of background noise from D1 to D2. Most background noise seems to be coming from direct $1.27 \mathrm{MeV}$ photons coming from the source as can be noted by the smallest peak seen in the spectrum for D1. A reduction of the background noise was able to be made by the placement of shielding directly between the source and D2, unfortunately our configuration did not allow for the same shielding to be placed between the source and D1 without a drastic reduction of overall counts. A 22 hour run was also done with no targets in and with our gate on to check for random coincidences, this was done to test reliability of real coincidences. In this case no coincidences were resolved.

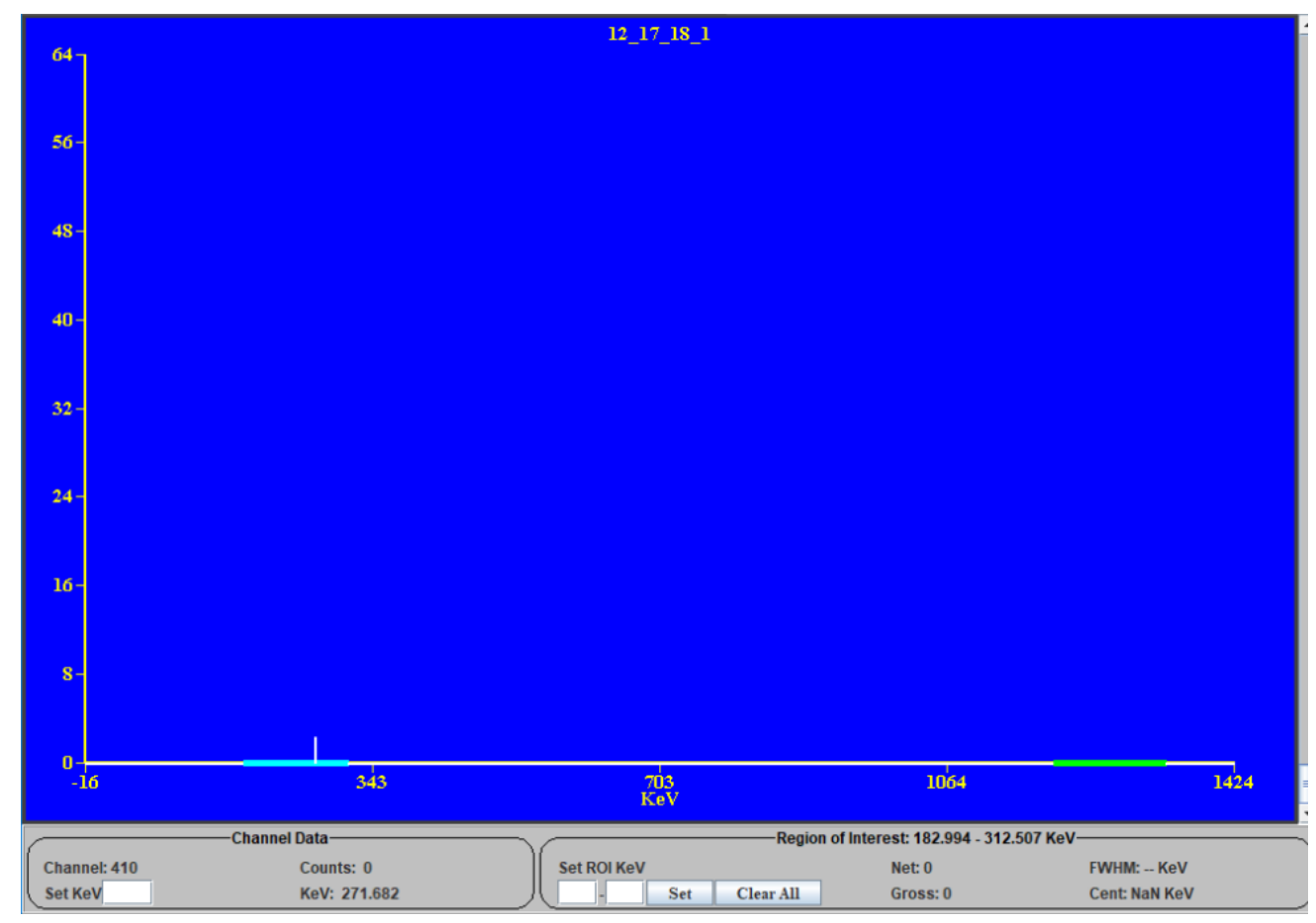

Figure 4: Target Out (Random Coincidence Check)

Finally coincidences were checked in the upright position and in the horizontal position. Coincidences were counted when any photon incident with D2 coincided with a photon within the range of our $0.255 \mathrm{MeV}$ photons detected by D1 and a spectrum was made by pulses from D2. Shown below is a coincidence spectrum in the upright position made over 
22 hours.

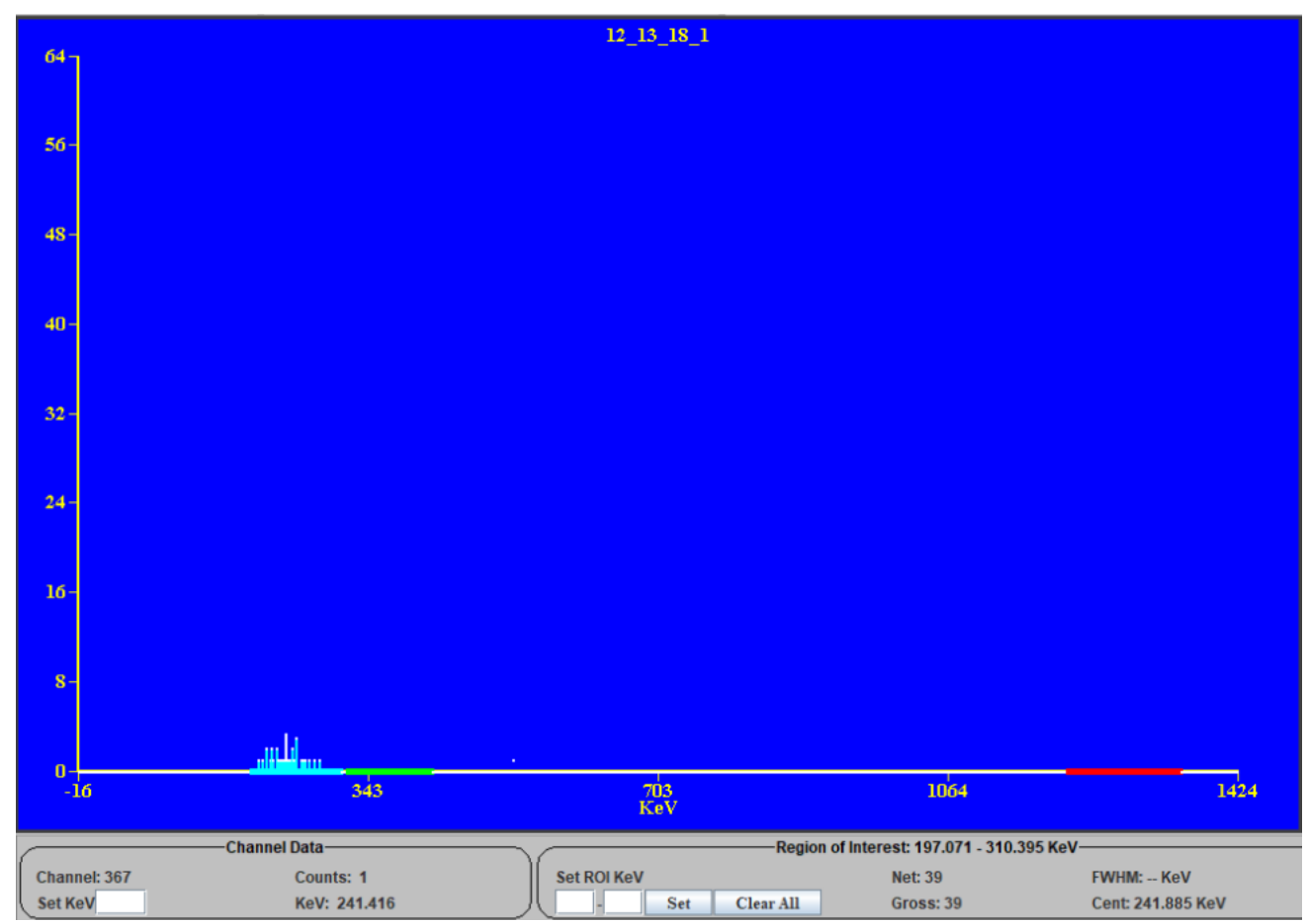

Figure 5: Vertical Detector Position Coincidence Detection

Here it would seem that most coincidences being detected are coming from real events as all but 1 registered detection fall within the expected energy range. Several runs were made with similar results. Unfortunately detection done in the horizontal position yielded similar results as can be seen below. 


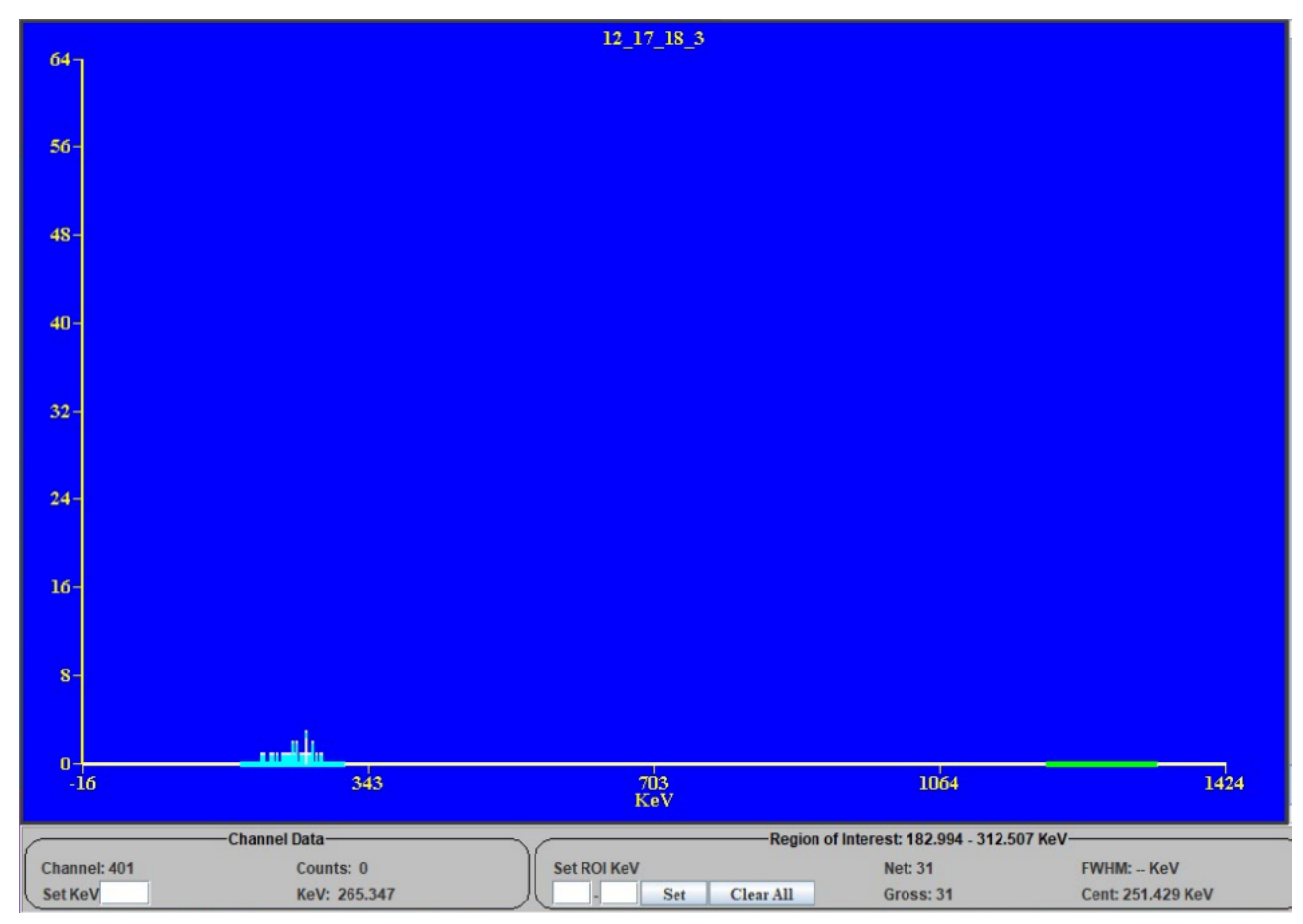

Figure 6: Horizontal Detector Position Coincidence Detection

The table below is a compilation of data from several runs done in both positions with all runs happening over a 22 hour period.

$\begin{array}{ccccc}\begin{array}{c}\text { Trial } \\ \text { Number }\end{array} & \begin{array}{c}\text { Vertical Position } \\ \text { Counts }\end{array} & \begin{array}{c}\text { uncer- } \\ \text { tainty }\end{array} & \begin{array}{c}\text { Horizontal Postion } \\ \text { Counts }\end{array} & \begin{array}{c}\text { uncer- } \\ \text { tainty }\end{array} \\ 1 & 39 & 6 & 31 & 6 \\ 2 & 44 & 7 & 36 & 6 \\ 3 & 43 & 7 & & \end{array}$

\section{Table 1: Coincidence Counts}

Though counts were obtained in both vertical and horizontal positions these preliminary results show a slight increase in counts for the vertical position which gives some hope that this increase can be accounted for by quantum entanglement. However more runs will need to be made to obtain enough data to be statistically viable.

\section{Conclusion}

There is some reason to believe we are seeing the effects of quantum entanglement in our study however we would like to see a more drastic reduction in counts for the horizontal 
position. We may be capable of doing this by reducing the size of our target so that we do not obtain as many coincidences from photons as a result of multiple scattering. However, by reducing the size of our target it will be necessary to have a more active source so that counts can be maintained. If a more active source is used this will most certainly increase background noise but it will as well increase our ability place our detectors further from the source giving us a better solid angle and increasing our ability to add shielding in the direct line between the source and the target. It would be useful as well to build a structure so that coincidence counts could be made at angles between our horizontal and vertical position as we expect this to follow a sine squared distribution. 